\title{
Application of Spike Sorting Algorithm to Neuronal Signals Originated from Boron Doped Diamond Micro-Electrode Arrays
}

\author{
Ondřej KLEMPÍŘn ${ }^{1}$, Radim KRUPIČKA ${ }^{1}$, Jan KRŮŠEK ${ }^{2}$, Ivan DITTERT ${ }^{2}$, Vladimíra \\ PETRÁKOVÁ ${ }^{1}$, Václav PETRÁK ${ }^{1}$, Andrew TAYLOR ${ }^{3}$ \\ ${ }^{1}$ Faculty of Biomedical Engineering, Czech Technical University in Prague, Kladno, Czech \\ Republic, ${ }^{2}$ Laboratory of Cellular Neurophysiology, Institute of Physiology of the Czech Academy \\ of Sciences, Prague, Czech Republic, ${ }^{3}$ Institute of Physics of the Czech Academy of Sciences, \\ Prague, Czech Republic
}

Received October 31, 2019

Accepted February 11, 2020

Epub Ahead of Print May 29, 2020

\section{Summary}

In this work we report on the implementation of methods for data processing signals from microelectrode arrays (MEA) and the application of these methods for signals originated from two types of MEAs to detect putative neurons and sort them into subpopulations. We recorded electrical signals from firing neurons using titanium nitride (TiN) and boron doped diamond (BDD) MEAs. In previous research, we have shown that these methods have the capacity to detect neurons using commerciallyavailable TiN-MEAs. We have managed to cultivate and record hippocampal neurons for the first time using a newly developed custom-made multichannel BDD-MEA with 20 recording sites. We have analysed the signals with the algorithms developed and employed them to inspect firing bursts and enable spike sorting. We did not observe any significant difference between BDD- and TiN-MEAs over the parameters, which estimated spike shape variability per each detected neuron. This result supports the hypothesis that we have detected real neurons, rather than noise, in the BDD-MEA signal. BDD materials with suitable mechanical, electrical and biocompatibility properties have a large potential in novel therapies for treatments of neural pathologies, such as deep brain stimulation in Parkinson's disease.

\section{Key words}

Microelectrode array • Boron doped diamond • Spike detection • Dimensionality reduction

\section{Corresponding author}

O. Klempiŕ, Faculty of Biomedical Engineering, Czech Technical University in Prague, nám. Sítná 3105, Kladno, 272 01, Czech Republic. E-mail: klempond@gmail.com

The use of microelectrode arrays (MEAs) is a widespread method for an in vitro neuronal network communication investigation, measuring spontaneous or stimulated activity (Pine 1980, Kunzinger 2019). MEA technology can be applied to any electrogenic tissue (i.e. central and peripheral neurons, heart cells and muscle cells), and is therefore capable of recording from hippocampal neurons (Jonsson et al. 2016). The electrode material is considered to be a key element for successful recording. The optimal material for electrodes should be mechanically stable, biocompatible, enable low-noise recording and allow easy surface chemical functionalization (Obien et al. 2015). Prototyping and employing a certain type of custom-made MEA to record and analyse electrical signals from a cultivated population can be a valuable alternative to well-known commercial devices in specific cases, e.g. mapping of rat auditory cortex (Lindovsky et al. 2018).

Various studies have shown that boron-doped diamond (BDD) materials with suitable mechanical, electrical and biocompatibility properties have a large potential in novel therapies for treatments of neural pathologies, such as deep brain stimulation (DBS) in 
Parkinson's disease, which is linked to abnormal extracellular neurochemical concentrations (Bennet et al. 2013, Rusinek et al. 2018, Tyszczuk-Rotko et al. 2019). Apart from excellent electrochemical properties and biocompatibility, BDD is a perspective material in terms of recording. Clinical DBS usually relies on predetermined stimulation parameters in an open-loop configuration. Results by (Bennet et al. 2016) suggest that BDD electrodes may be the solution for chronic DBS implantation, which could support long-term investigation of neurochemical responses in humans. This could potentially lead to close-loop in DBS and utilize neurochemical feedback to dynamically adjust stimulation parameters.

Furthermore, previous studies already demonstrated that BDD doping significantly improves the characteristics of sensing electrical signals (Matsubara et al. 2016, Zehani et al. 2015). Demonstrated application of BDD-MEAs in the biomedical field include all diamond MEAs for recording of electrogenic cells (Maybeck et al. 2014) and nanostructured BDD for MEA neural interfacing (Piret et al. 2015). However, these efforts have focused on the fabrication route challenges rather than on actual application of MEAs for recording from biological samples.

The processing of extracellular signals is a computationally and conceptually complex task with multiple sources of errors, which can affect downstream data interpretation. It is challenging to solve the problem of automatic detection of action potentials satisfactorily for the following reasons:

1) Spike shapes and background noise are different for a neuron.

2) Attenuated action potentials from distant neurons can also penetrate into the recorded signal, it is difficult to clearly determine what we consider to be a relevant spike and what is noise. Indeed, often even two experts disagree on the makeup of a single recording.

3) Signal to noise ratio may be very low in some signals (Wilson and Emerson 2002).

The current study builds upon our previous work (Klempir et al. 2019, Krusek et al. 2019). In this short communication, we present BDD-MEA signals and application of quantitative signal processing.

The whole microelectrode recording pipeline includes multiple steps ranging from initial sample preparation to analysis and interpretation. Extracellular neural activity was acquired by two MEA devices independently. For experiments, we used a commercial
60 channel titanium nitride (TiN) microelectrode array (USB-ME64-System by Multi Channel Systems MCS $\mathrm{GmbH}$, Reutlingen, Germany) as a reference platform $(210 \times 210 \times 35 \mathrm{~mm}) .60 \mathrm{TiN}$ electrodes are arranged in $8 \times 8$ grid, with a sampling rate of up to $50 \mathrm{kHz}$ (Fig. 1C). The layout of the fabricated BDD-MEA is comparable to the commercial MEA (Fig. 1A), but due to technical reasons, the number of electrodes was reduced to 20 (Fig. 1D). Figure 1B corresponds to the distribution of contacts in Figure 1D with 20 electrodes. The visible imperfections, but still satisfying electrode alignment (Fig. 1B), is related to the ink-jet printing technology used to produce localized nano-diamond seeding prior to BDD growth, which still requires optimization. A total of 4 BDD chambers have been prepared with this technology.

Deposition of BDD is realized using a linear antenna microwave plasma enhanced chemical vapor deposition system using conditions described in (Taylor et al. 2018) to produce conductive diamond. Chip preparation included the fixation of a glass ring using Sylgard elastomer and sterilization. Chemical treatment of neuroelectrodes was performed by treating material with selected cationic polymer (poly-L-lysine). The role of this polymer is to enhance cell adherence to the surface and increase attachment of cells. This method builds upon previous results of molecular functionalization of planar nanocrystalline and porous nanostructured diamond to form an interface with newborn and adult neurons, which has been described in (Krusek et al. 2019, Taylor et al. 2018).

Adult hippocampal neurons of male rats of the Wistar strain were used in this experiment and were handled in accordance with law No. 246/1992 of the Czech Republic and the current Guidelines for the Treatment of Laboratory Animals (EU Guidelines 86/609/EEC).

The reference TiN-MEA dataset contains 6 continuous measurements of spontaneous activity of 60 channels. Signal time series included quiet spots, bursting and isolated spikes (Fig. 1E). In the case of BDDMEA, the dataset contains 9 continuous measurements of spontaneous activity of 20 channels. Each electrode corresponds to an input time series channel and the recorded files had a length of approximately $6 \mathrm{~min}$ with a sampling rate of $20 \mathrm{kHz}$. Initial measurements were started immediately after the preparation of neural cultures with spiking activity becoming apparent after 1 week. Spikes were recorded for 14 days under the same conditions. A raw BDD-MEA electrophysiological signal of the neurons is presented in Figure 1F. 

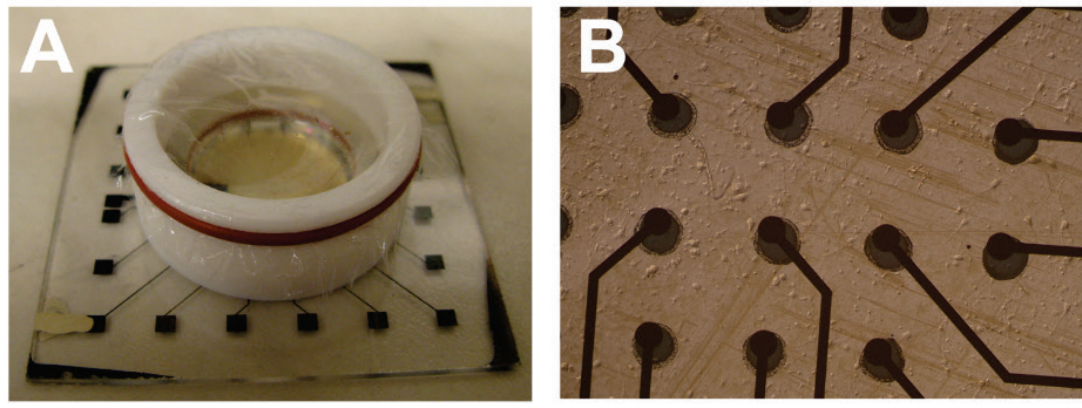

C

\begin{tabular}{c|c|c|c|c|c|c|c} 
& 21 & 31 & 41 & 51 & 61 & 71 & \\
\hline 12 & 22 & 32 & 42 & 52 & 62 & 72 & 82 \\
\hline 13 & 23 & 33 & 43 & 53 & 63 & 73 & 83 \\
\hline 14 & 24 & 34 & 44 & 54 & 64 & 74 & 84 \\
\hline $\mathrm{G}$ & 25 & 35 & 45 & 55 & 65 & 75 & 85 \\
\hline 16 & 26 & 36 & 46 & 56 & 66 & 76 & 86 \\
\hline 17 & 27 & 37 & 47 & 57 & 67 & 77 & 87 \\
\hline & 28 & 38 & 48 & 58 & 68 & 78 &
\end{tabular}

D

\begin{tabular}{l|l|l|l|l|l} 
& 33 & 41 & 51 & 63 & \\
\hline 24 & 23 & 31 & 61 & 73 & 74 \\
\hline 25 & 26 & 38 & 68 & 76 & 75 \\
\hline & 36 & 48 & 58 & 66 &
\end{tabular}

Fig. 1. Signal acquisition of neuronal activity on MEAs. (A) BDD-MEA with glass ring and gas permeable membrane lid. (B) BDD-MEA with visible hippocampal neurites and neurons growth across electrodes. (C) Reference TiN-MEA electrode distribution. ( $\mathrm{G}=$ ground). (D) BDD TiNMEA electrode distribution. (E) Raw recording of spontaneous hippocampal neuronal activity recorded in vitro from TiN-MEA. (F) Raw recording of spontaneous hippocampal neuronal activity recorded in vitro from BDDMEA. Peaks represent a spike, bursting activity is also evident. The noise level is around $\pm 5 \mu \mathrm{V}$ while signal amplitudes are from $\pm 15 \mu \mathrm{V}$.
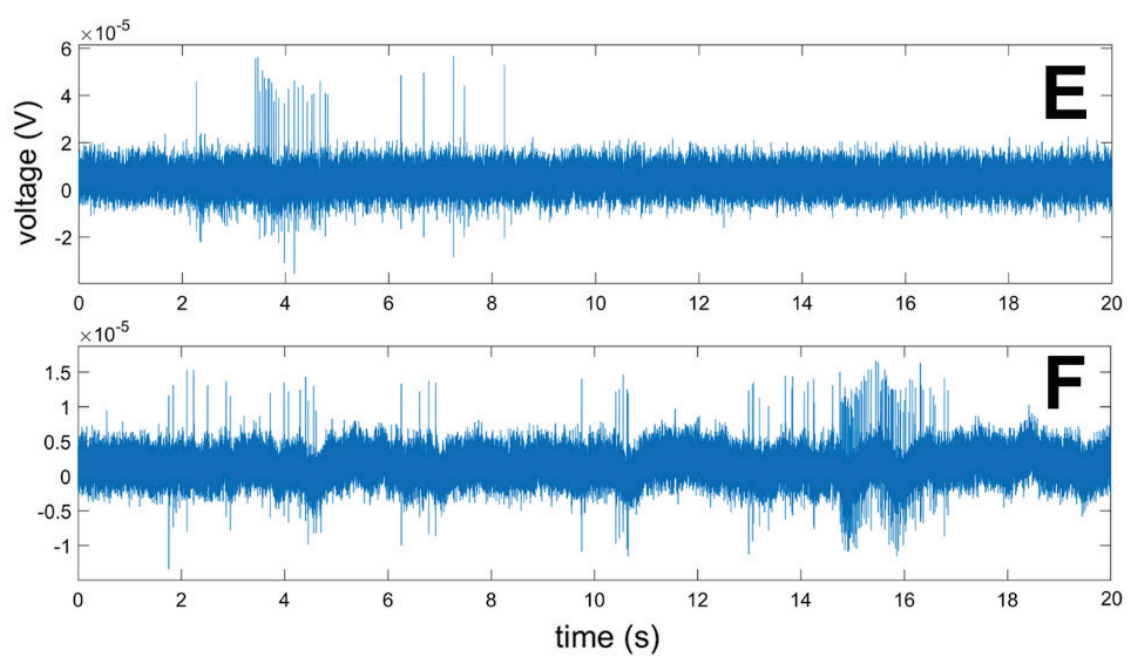

In order to obtain individual spikes from microelectrode recordings, we recently implemented an automated analytical pipeline (Klempir et al. 2019): containing signal filtering, spike detection, spike sorting and individual spike train features computation. The proposed pipeline in Matlab (MathWorks, Natick, Massachusetts, USA) was applied to automate the extraction of neuron signals from both types of MEAs.

Signals were band-pass filtered (Butterworth $2^{\text {nd }}$ ) over the $300-3000 \mathrm{~Hz}$ range. The number and time location of spikes were detected by the spike detection process. Since detection trade-off is related to the signal to noise ratio of recordings, an amplitude WaveClus detector was used for spike detection (Table 1). The WaveClus algorithm is an unsupervised spike detection and sorting tool, which has performed reasonably well on single-channel micro-recordings for both positive and negative spike polarities (https:/github.com/csn- le/wave_clus). To capture the time courses of truly positive spikes, we used a threshold cut-off (Thr) of 5 times the standard deviation of the input signal noise (https://github.com/dbridges/mea-tools).

$$
T h r=5 \sigma_{n} \quad \sigma_{n}=\text { median }\left\{\frac{\mid \text { filtered signal } \mid}{0.6745}\right\}
$$

The number of samples corresponding to the minimum refractory period was considered as 36 (i.e. $1.8 \mathrm{~ms}$ ). Each detected spike bounded by a range of the pre- and post-event data points was modulated by a cubic spline curve and 20 equidistant shape-based features were computed (Table 1). Numerically, each row (i.e. spike waveform shape) in the dataset is represented by a vector of 20 numbers determining the neural morphologies and the distances and orientations relative to the recording electrode. 
Table 1. WaveClus detector: an overview of the set hyperparameters.

\begin{tabular}{lc}
\hline Hyperparameter & value \\
\hline Minimum refractory period (data points) & 36 \\
Number of pre-event data points stored & 10 \\
Number of post-event data points stored & 10 \\
Spike waveform interpolation & Cubic spline \\
Maximum detection threshold & $30 \sigma_{\text {signal }}$ \\
\hline
\end{tabular}

The main output of the detection function from the whole duration and all channels contains indexes of detected peak positions, threshold amplitudes and associated waveforms. For downstream analysis, we included only active channels. The channel must detect at least 20 spikes in order for the channel to be considered active.

For data exploration, we investigated transformations of individual spike waveforms through embedding into a lower dimension. We anticipated extending Principal Component Analysis (PCA) with UMAP (Uniform Manifold Approximation and Projection) and replacing the subsequent clustering K-means algorithm with Louvain method. Namely, the current pipeline contains clustering and identification of waveforms types on the principle of Scanpy package in Python (Wolf et al. 2018), which stands out in its ability to identify subpopulations in single cell data. We reduced the dimensionality of the data by running PCA, which reveals the main axes of variation and denoises the waveform data. Then we computed the neighborhood graph matrix using the 5 PCAs representation of the data matrix based on similarity of waveforms. We embedded the neighborhood graph $(\mathrm{n}=10)$ in 2 dimensions using UMAP with ScanPy default settings. Finally, the unsupervised Louvain graph-clustering method directly clusters the neighborhood graph of waveforms to sort them automatically into subpopulations.

The level of spike-to-spike variability was further evaluated for the sorted spikes. We implemented the method published by (Stratton et al. 2012). Spike variability was calculated so that a variability of 0 indicated that every spike waveform from a given neuron was exactly the same (i.e. no variability) and that variability approached 1 as noise began to dominate the waveforms (i.e. spike shapes became random). Each spike in a unique putative neuron was then compared to the mean spike for that neuron using the dot product (cosine distance). The mean of the distribution of dot product values for all spikes provided a measure of the variability from spike to spike, independent of any amplitude changes (since the spike vectors were normalized) (Stratton et al. 2012).

We continued by checking for consistency between spike-to-spike variability and a second alternative variability estimation approach. For each putative neuron, the median from absolute values of individual coefficients of variation (CV) across individual 20 shape-based features was calculated. Distributions with a CV found to be less than 1 were considered to be low-variance, whereas those with a $\mathrm{CV}$ higher than 1 were considered to be high variance.

As voltages and spike-to-spike parameters were not found to be normally distributed by the one-sample Shapiro-Wilk test, statistical comparisons were performed using a non-parametric Mann-Whitney test. The correlation was assessed using the Spearman correlation coefficient, which measures monotonic relationship. The statistical significance was determined as $\mathrm{p}=0.05$.

Detected waveforms across all the measurements ( $>20$ spikes on recording) were projected into a reduced UMAP space, as can be seen in Fig. 2E. Evaluation of voltage waveforms collected from BDD-MEA $(n=10403)$ vs. commercially available TiN-MEA $(\mathrm{n}=11346)$ reveals that they differ mostly in amplitude values $(p<0.05)$. Significantly smaller amplitudes were observed in BDDMEA. The differences in amplitudes could be caused by HW and may not reflect a difference in physiology. Furthermore, we determined clusters using the unsupervised Louvain method, which found 14 waveform subpopulations (Fig. 2C). By assigning waveforms to subpopulations, in total 35 putative neurons in TiN-MEA and 31 putative neurons in BDD-MEA were found. Examples of variability in neuronal shapes for both types of MEAs are illustrated in Figure 2B. 

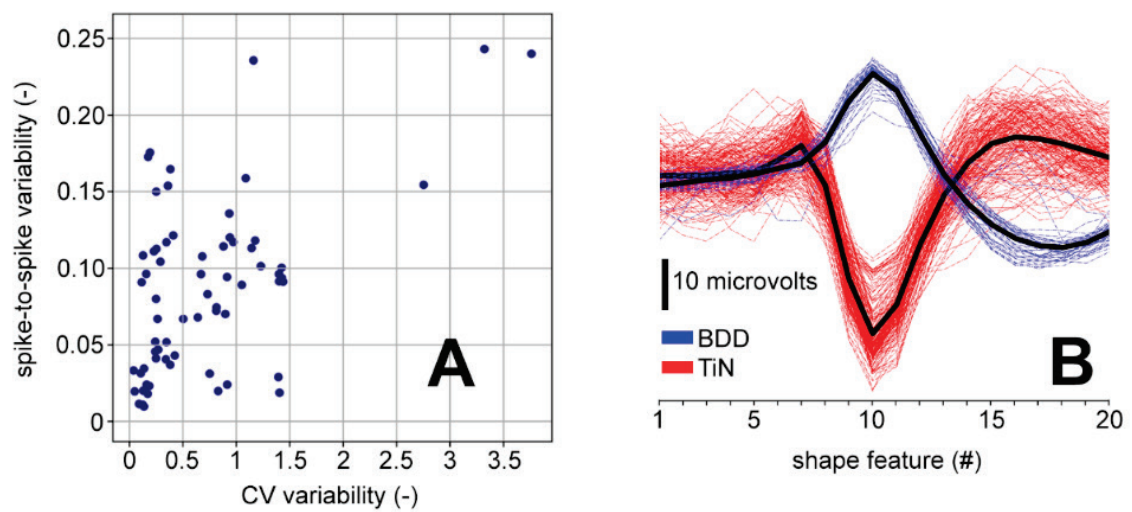

Fig. 2. (A) Scatter plot representing relationship between both variability estimations. (B) Example spikes from both types of MEAs. The mean spikes (black) and the spikes of those means. (C) UMAP plot visualizing cluster assignments of waveforms, which are defined by Louvain clustering and denoted as distinct colors. (D) Distribution of spike-to-spike variability via boxplots. (E) Coloring by individual measurements.
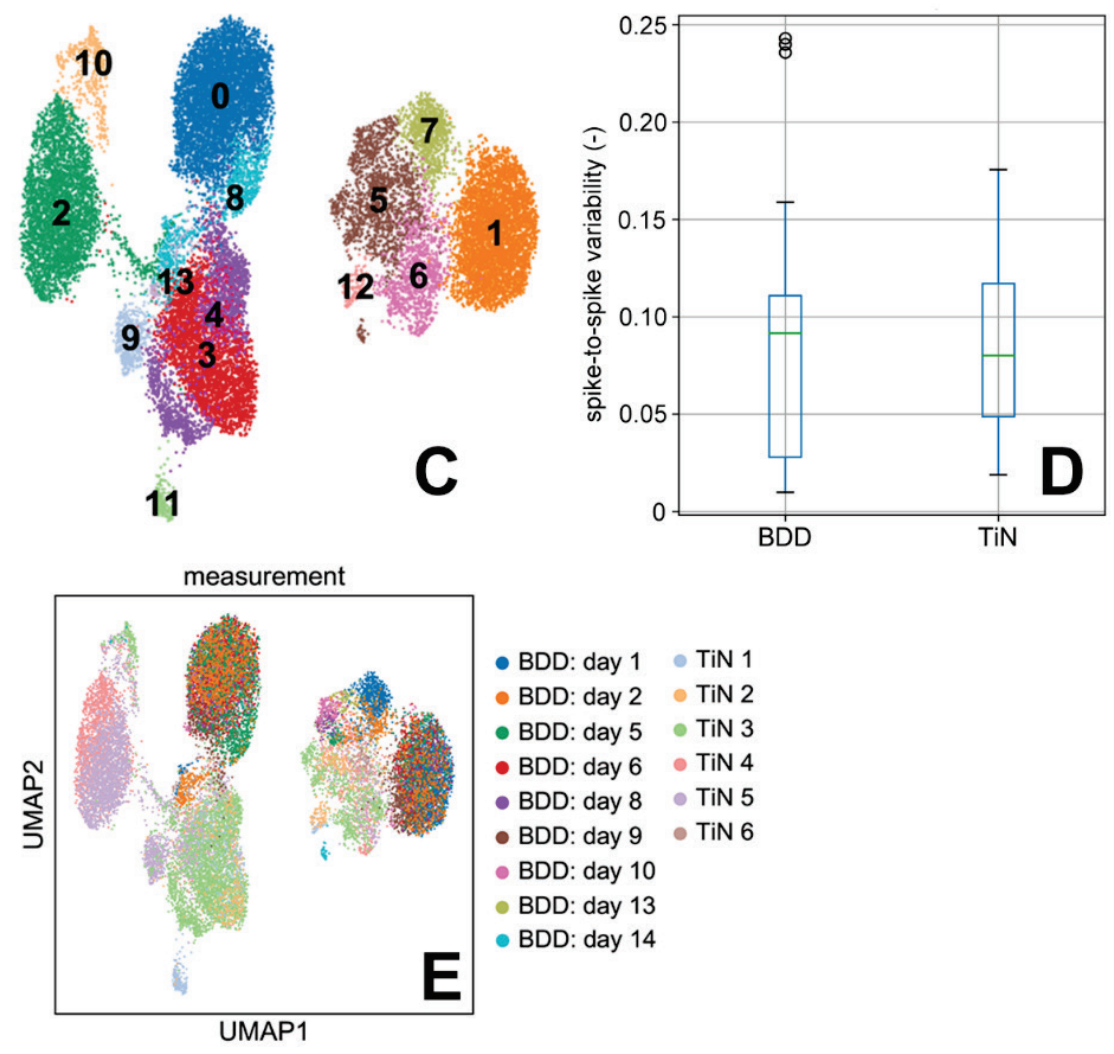

Statistical testing did not show any significant difference $(\mathrm{p}=0.27)$ in the spike-to-spike variability in BDD-MEA ( $\mathrm{n}=31)$ vs. TiN-MEA $(\mathrm{n}=35)$, which supports the hypothesis that we detect real neurons rather than noise in the BDD signals. With the same $\mathrm{Thr}=5$, BDD-MEA has a slightly higher level of variability $(0.09 \pm(\mathrm{SD}) 0.07)$ against $(0.09 \pm(\mathrm{SD}) 0.05)$ for TiNMEA (Fig. 2D). Scatter plot demonstrated significant consistency $(\mathrm{rho}=0.43 ; \mathrm{p}<0.001)$ of both methods for variability estimation (Fig. 2A). Given the $\mathrm{CV}$ variability could be sensitive to outliers, the value of correlation coefficient is not representing a completely tight relationship. Overall, the detection method proves to be feasible for detection from various biological samples.

Apart from the clear BDD spike activity, we have noticed some other activities on some of the electrodes whose origin we do not understand. Either they are minor events covered in noise, but by shape they could be normal activity, only recorded from a greater distance, or with a worse contact. We even saw events of a completely different shape, whose origin is unknown. Sometimes rectangular events were recorded, which look like neural channel openings. However, their length was several times the length of the normal spike and were suppressed by filtering.

Comparing the performance of UMAP with five other tools, UMAP should provide the fastest run times, highest reproducibility and the most meaningful organization of cell clusters (Becht et al. 2019). The most notable is that UMAP, like t-distributed Stochastic 
Neighbor Embedding, does not completely preserve density and can also create clusters that are not actually present, resulting in a finer clustering than is necessarily present in the data. On the other hand, the UMAP visualisations with apparent separation of data could facilitate discovery of rare spikes population, which would not be possible without upgrading our pipeline.

Finding action potentials in a signal based on their shape, in other words, template matching, is another method of spike detection. It uses a manually created database of spike shapes, with which parts of the signal are continuously compared. With this method, it is possible to achieve much better results than simply thresholding the signal (Kim and McNames 2007). However, as each neuron or group of neurons has a different characteristic spike shape, use of this method requires manual creation of a database of spike shapes for each analysed signal. Therefore, this method is unsuitable for automatic spike detection.

The BDD-MEA density of the electrodes is lower compared to the TiN-MEA, which leads to a lower resolution of the electrophysiological picture of the neuronal network and therefore to a lower statistical significance with regard to the interpretation of the network functionality. To record all 20 BDD-MEA channels at $20 \mathrm{kHz}$ generates a huge amount of data. A six-minute recording creates a file, which is $1 \mathrm{~GB}$ in size. In order to analyze this data, high demands must be placed on laboratory hardware and software (Kunzinger 2019).

We ultimately aim at the development of BDD MEA for dual recording, which will employ BDD based micro-probes positioned within close proximity of a firing neuron on an MEA electrode for simultaneous recording of electrical and chemical activity. As a first step towards this goal, we have measured cyclic voltammograms recorded on BDD micro-probe demonstrating dopamine detection under simulation conditions, i.e. controlled release into cell culture dishes to characterize micro-probe sensitivity (Baluchova et al. 2019).

In conclusion, we have presented extracellular recordings, from cultures of hippocampal neurons on BDD-MEAs over a period of weeks allowing analysis of network development. Previous research showed only signals without closer analysis of BDD-MEA spikes (Piret et al. 2015).

\section{Conflict of Interest}

There is no conflict of interest.

\section{Acknowledgements}

The work was supported by the Grant Agency of the Czech Republic, No. 17-15319S (GAČR).

\section{References}

BALUCHOVA S, TAYLOR A, MORTET V, SEDLAKOVA S, KLIMSA L, KOPECEK J, HAK O, SCHWARZOVAPECKOVA K: Porous boron doped diamond for dopamine sensing: Effect of boron doping level on morphology and electrochemical performance. Electrochim Acta 327: 135025, 2019. https://doi.org/10.1016/j.electacta.2019.135025

BECHT E, MCINNES L, HEALY J, DUTERTRE CA, KWOK IWH, NG LG, GINHOUX F, NEWELL EW: Dimensionality reduction for visualizing single-cell data using UMAP. Nat Biotechnol 37: 38-44, 2019. https://doi.org/10.1038/nbt.4314

BENNET KE, LEE KH, KRUCHOWSKI JN, CHANG SY, MARSCH MP, VAN ORSOW AA, PAEZ A, MANCIU FS: Development of conductive boron-doped diamond electrode: a microscopic, spectroscopic, and voltammetric study. Materials (Basel) 6: 5726-5741, 2013. https://doi.org/10.3390/ma6125726

BENNET KE, TOMSHINE JR, MIN HK, MANCIU FS, MARSH MP, PAEK SB, SETTELL ML, NICOLAI EN, BLAHA CD, KOUZANI AZ, CHANG SY, LEE KH: A diamond-based electrode for detection of neurochemicals in the human brain. Front Hum Neurosci 10: 102, 2016. https://doi.org/10.3389/fnhum.2016.00102

FERREA E, MACCIONE A, MEDRIHAN L, NIEUS T, GHEZZI D, BALDELLI P, BENFENATI F, BERDONDINI L: Large-scale, high-resolution electrophysiological imaging of field potentials in brain slices with microelectronic multielectrode arrays. Front Neural Circuits 6: 8, 2012. https://doi.org/10.3389/fncir.2012.00080 
JONSSON A, INAL S, UGUZ I, WILLIAMSON AJ, KERGOAT L, RIVNAY J, KHODAGHOLY D, BERGGREN $\mathrm{M}$, BERNARD $\mathrm{CH}$, MALLIARAS GG, SIMON DT: Bioelectronic neural pixel: Chemical stimulation and electrical sensing at the same site. Proc Natl Acad Sci U S A 113: 9440-9445, 2016. https://doi.org/10.1073/pnas.1604231113

KIM S, MCNAMES J: Automatic spike detection based on adaptive template matching for extracellular neural recordings. J Neurosci Meth 165: 165-174, 2007. https://doi.org/10.1016/j.jneumeth.2007.05.033

KLEMPIR O, KRUPICKA R, PETRAKOVA V, KRUSEK J, DITTERT I, TAYLOR A: Automated neurons recognition and sorting for diamond based microelectrode arrays recording: A feasibility study. In: World Congress on Medical Physics and Biomedical Engineering 2018. LHOTSKA L, SUKUPOVA L, LACKOVIC I, IBBOTT G (eds), Springer, Singapore, 2019, pp 281-286. https://doi.org/10.1007/978-981-10-9038-7_52

KRUSEK J, DITTERT I, SMEJKALOVA T, KORINEK M, GOTTFRIEDOVA K, FREISLEBENOVA H, NEUHOFEROVA E, KLIMSA L, SEDLAKOVA S, TAYLOR A, MORTET V, PETRAK V, PETRAKOVA $\mathrm{V}$ : Molecular functionalization of planar nanocrystalline and porous nanostructured diamond to form an interface with newborn and adult neurons. Phys Status Solidi 256: 1800424, 2019. https://doi.org/10.1002/pssb.201800424

KUNZINGER S: Signal recording of 3D neurospheres on high-resolution CMOS MEA platform. In: Proceedings of the International Student Scientific Conference Poster - 23/2019. HUSNIK L (ed.), Czech Technical University in Prague, Prague, 2019, pp 33-35.

LINDOVSKY J, PYSANENKO K, POPELAR J, SYKA J: Fast tonotopy mapping of the rat auditory cortex with a custom-made electrode array. Physiol Res 67: 993-998, 2018. https://doi.org/10.33549/physiolres.933835

MACCIONE A, GANDOLFO M, ZORDAN S, AMIN H, DI MARCO S, NIEUS T, ANGOTZI GN, BERDONDINI L: Microelectronics, bioinformatics and neurocomputation for massive neuronal recordings in brain circuits with large scale multielectrode array probes. Brain Res Bull 119: 118-126, 2015. https://doi.org/10.1016/j.brainresbull.2015.07.008

MATSUBARA T, UJIE M, YAMAMOTO T, AKAHORI M, EINAGA Y, SATO T: Highly sensitive detection of influenza virus by boron-doped diamond electrode terminated with sialic acid-mimic peptide. Proc Natl Acad Sci U S A 113: 8981-8984, 2016. https://doi.org/10.1073/pnas.1603609113

MAYBECK V, EDGINGTON R, BONGRAIN A, WELCH JO, SCORSONE E, BERGONZO P, JACKMAN RB, OFFENHAUSSER A: Boron-doped nanocrystalline diamond microelectrode arrays monitor cardiac action potentials. Adv Healthc Mater 3: 283-289, 2014. https://doi.org/10.1002/adhm.201300062

OBIEN ME, DELIGKARIS K, BULLMANN T, BAKKUM DJ, FREY U: Revealing neuronal function through microelectrode array recordings. Front Neurosci 8: 423, 2015. https://doi.org/10.3389/fnins.2014.00423

PINE J: Recording action potentials from cultured neurons with extracellular microcircuit electrodes. J Neurosci Meth 2: 19-31, 1980. https://doi.org/10.1016/0165-0270(80)90042-4

PIRET G, HEBERT C, MAZELLIER JP, ROUSSEAU L, SCORSONE E, COTTANCE M, LISSORGUES G, HEUSCHKEL MO, PICAUD S, BERGONZO P, YVERT B: 3D-nanostructured boron-doped diamond for microelectrode array neural interfacing. Biomaterials 53: 173-183, 2015. https://doi.org/10.1016/j.biomaterials.2015.02.021

RUSINEK CA, GUO Y, RECHENBERG R, BECKER MF, PURCELL E, VERBER M, MCKINNEY C, LI W: All-diamond microfiber electrodes for neurochemical analysis. J Electrochem Soc 165: 3087-3092, 2018. https://doi.org/10.1149/2.0141812jes

STRATTON P, CHEUNG A, WILES J, KIYATKIN E, SAH P, WINDELS F, ARABZADEH E: Action potential waveform variability limits multi-unit separation in freely behaving rats. PLoS One 7: e38482, 2012. https://doi.org/10.1371/journal.pone.0038482

TAYLOR A, ASHCHEULOV P, HUBIK P, KLIMSA L, KOPECEK J, REMES Z, VLCKOVA ZIVCOVA Z, REMZOVA M: Precursor gas composition optimisation for large area boron doped nano-crystalline diamond growth by MW-LA-PECVD. Carbon 128: 164-171, 2018. https://doi.org/10.1016/j.carbon.2017.11.063

TYSZCZUK-ROTKO K, JAWORSKA I, JEDRUCHNIEWICZ K: Application of unmodified boron-doped diamond electrode for determination of dopamine and paracetamol. Microchem J 146: 664-672, 2019. https://doi.org/10.1016/j.microc.2019.01.064 
WILSON SB, EMERSON R: Spike detection: a review and comparison of algorithms. Clin Neurophysiol 113: 1873-1881, 2002. https://doi.org/10.1016/s1388-2457(02)00297-3

WOLF FA, ANGERER P, THEIS FJ: SCANPY: large-scale single-cell gene expression data analysis. Genome Biol 19: 15, 2018. https://doi.org/10.1186/s13059-017-1382-0

ZEHANI N, FORTGANG P, SADDEK LACHGAR M, BARAKET A, ARAB M, DZYADEVYCH SV, KHERRAT R, JAFFREZIC-RENAULT N: Highly sensitive electrochemical biosensor for bisphenol A detection based on a diazonium-functionalized boron-doped diamond electrode modified with a multi-walled carbon nanotubetyrosinase hybrid film. Biosens Bioelectron 74: 830-835, 2015. https://doi.org/10.1016/j.bios.2015.07.051 